\title{
Contribution of Double-Logarithmic Pomeron to structure function $F_{1}$
}

\section{B.I. Ermolaev*}

Ioffe Physico-Technical Institute, 194021 St.Petersburg, Russia

E-mail: boris.ermolaevamail.cern.ch

\section{S.I. Troyan}

St.Petersburg Institute of Nuclear Physics, 188300 Gatchina, Russia

E-mail: serqei.troyandthd.pnpi.spb.ru

Pomeron was introduced in the framework of the phenomenological Regge theory. It governs the high-energy asymptotics of various hadronic processes and the small- $x$ behavior of $F_{1}$ in particular. The best-known contribution to the QCD Pomeron comes from the BFKL equation which sums Leading Logarithmic (LL) contributions, i.e. the single-logarithmic (SL) contributions $\sim\left(\alpha_{s} \ln (1 / x)\right)^{n}$ multiplied by the overall factor $1 / x$. The high-energy asymptotics of this resummation is known as the BFKL Pomeron. It predicts that at asymptotically small $x, F_{1} \sim x^{-1-\Delta}$ where $\Delta$ is the intercept of the BFKL Pomeron. In contrast, we calculate $F_{1}$ in the DoubleLogarithmic Approximation (DLA), accounting for contributions $\sim\left(\alpha_{s} \ln ^{2}(1 / x)\right)^{n}$ together with double-logs of $Q^{2}$ to all orders in $\alpha_{s}$. Such terms are not accompanied by the overall factor $1 / x$, so the small- $x$ asymptotics of their sum is $\sim x^{-\Delta_{D L}}$ which looks negligibly small compared to the BFKL exponent $1+\Delta$. By this reason the DL contribution to Pomeron was offhandedly ignored by the HEP community. However, we demonstrate that the intercept $\Delta_{D L}$ proves to be so large that its value compensates for the lack of $1 / x$. This makes the DL Pomeron and BFKL Pomeron be equally important. Therefore, DL Pomeron should participate in theoretical analysis whenever the BFKL Pomeron is accounted for.

XXVII International Workshop on Deep-Inelastic Scattering and Related Subjects - DIS2019 8-12 April, 2019

Torino, Italy

\footnotetext{
* Speaker.
} 


\section{Introduction}

Straightforward theoretical investigation of DIS required QCD calculations at both high and low energies. While the former can be done with means of Perturbative QCD, the latter is unavailable at present. Its impact is mimicked by assumption of QCD factorization which represents the DIS hadronic tensor $W_{\mu v}$ though convolutions of perturbative and non-perturbative components. The tensor $W_{\mu \nu}$ is parameterized by the DIS structure functions, each of them is also represented though convolutions. For instance, $F_{1}$ can be represented as follows:

$$
F_{1}=T_{q} \otimes \Phi_{q}+T_{g} \otimes \Phi_{g}
$$

where $T_{q}$ and $T_{g}$ are the perturbative components. They correspond to DIS off a quark and gluon respectively and are calculated in the framework of Perturbative QCD. In contrast, the initial parton distributions $\Phi_{q}$ and $\Phi_{g}$ are essentially non-perturbative objects. They are chosen on basis of phenomenological considerations, depending on the form of QCD factorization. There are known three forms of QCD factorization in the literature but here we consider $F_{1}$ in Collinear Factorization only.

The standard theoretical instrument for description of $T_{q}$ and $T_{g}$ at large $x$ and large $Q^{2}$ is DGLAP[W]. DGLAP controls the $Q^{2}$-evolution of $T_{q, g}$, while the contributions $\sim \ln ^{n}(1 / x)$ are left accounted in few first orders in $\alpha_{s}$ only. Being unimportant at large $x$, these contributions become large at low $x$, where their total resummation is essential. One example of such a resummation is done with the BFKL equation[D]. It neglects $T_{q}$ and sums the series of single-logarithmic contributions to $T_{g}$ accompanied by the overall factor $1 / x$ to all orders in $\alpha_{s}$ :

$$
(1 / x)\left[1+c_{1} \alpha_{s} \ln (1 / x)+c_{2}\left(\alpha_{s} \ln (1 / x)\right)^{2}+\ldots\right],
$$

with $c_{n}$ being numerical factors. The asymptotics of the sum in Eq. ([L2) is called the BFKL Pomeron. It governs the small- $x$ asymptotics of $F_{1}$ :

$$
F_{1}^{(B F K L)} \sim x^{-1-\Delta}
$$

when $x \rightarrow 0$. The intercept $\Delta$ is calculated in LO in Ref. [0] and in NLO in Refs. [B], 田].

Another example of total resummations of the contributions to $F_{1}$ essential at small $x$ is the Double-Logarithmic Approximation (DLA) which accounts for the following series:

$$
1+c_{1}^{\prime} \alpha_{s} \ln ^{2}(1 / x)+c_{2}^{\prime}\left(\alpha_{s} \ln ^{2}(1 / x)\right)^{2}+\ldots
$$

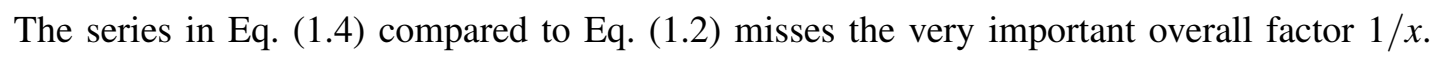
This was regarded as an undisputable evidence to neglect (ㄷ.4) without performing any calculations. However, in Ref. [可] we demonstrated that this verdict was false and as a matter of fact the contribution of the DL series (ㄷ.4) was quite essential. We demonstrate below how to compose and solve the Infra-Red Evolution Equations (IREEs) for $T_{q, g}$, and consider their small- $x$ asymptotics. 


\section{Evolution Equations for $T_{q, g}$}

We start with calculating the invariant amplitudes $A_{q, g}$ of Compton scattering related to $T_{q, g}$ by Optical theorem:

$$
T_{q}=\frac{1}{\pi} \mathfrak{I} A_{q}, \quad T_{g}=\frac{1}{\pi} \mathfrak{I} A_{g} .
$$

Throughout the paper we will use for amplitudes $T_{q, g}$ as well as for other amplitudes the asymptotic form of the Sommerfeld-Watson transform frequently addressed as the Mellin transform. This transform is:

$$
A_{q, g}\left(w, Q^{2} / \mu^{2}\right)=\int_{-1 \infty}^{1 \infty} \frac{d \omega}{2 \imath \pi}\left(w / \mu^{2}\right)^{\omega} \xi^{(+)}(\omega) F_{q, g}(\omega, y),
$$

where we have introduced the logarithmic variable

$$
y=\ln \left(Q^{2} / \mu^{2}\right)
$$

and the positive signature factor

$$
\xi^{(+)}(\omega)=-\left(e^{-\imath \pi \omega}+1\right) / 2 .
$$

In Eq. (2.2]) $w=2 p q$, with $p$ being the external parton momenta, and $\mu$ is the factorization scale. We will calculate $A_{q, g}$ with composing and solving Infra-Red Evolution Equations (IREEs). This method was suggested by L.N. Lipatov. The history and detailed description of this method can be found in Ref. [目]. The method stems from the observation made by V.N. Gribov that the partons with minimal transverse momenta can be factorized with DL accuracy. In regard of $F_{q, g}$, the technical steps in the simplest version of the method are as follows: First, neglect quark masses in order to treat virtual quarks and gluons equally. It makes all DL contribution to be IR divergent. Second, introduce the infra-red (IR) cut-off $\mu$ in the transverse momentum space to regulate the IR divergences. Third, trace the evolution of $A_{q, g}$ with respect to $\mu$ choosing the partons with minimal transverse momentum $k_{\perp}$ and use factorization of the $k_{\perp}$-dependent parts of $A_{g, g}$. Applying the operator $-\mu^{2} \partial / \partial \mu^{2}$ to Eq. (L2) , we obtain the L.h.s. of the IREEs. The r.h.s. is formed by convolutions of the factorized amplitudes $A_{q, g}$. In the $\omega$-space the IREEs are (see Ref. [[]] for detail):

$$
\begin{aligned}
& \partial F_{q}(\omega, y) / \partial y+\omega F_{q}(\omega, y)=1 /\left(8 \pi^{2}\right)\left[F_{q}(\omega, y) f_{q q}(\omega)+F_{g}(\omega, y) f_{g q}(\omega)\right], \\
& \partial F_{g}(\omega, y) / \partial y+\omega F_{g}(\omega, y)=1 /\left(8 \pi^{2}\right)\left[F_{q}(\omega, y) f_{q g}(\omega)+F_{g}(\omega, y) f_{g g}(\omega)\right] .
\end{aligned}
$$

Eq. (ㄷ.5) involves new objects: the parton-parton amplitudes $f_{r r^{\prime}}$, with $r, r^{\prime}=q, g$. They describe the parton-parton scattering. IREEs for them should be constructed independently. Once $f_{r r^{\prime}}$ are known, a general solution to Eq. ([2.5) can be obtained with regular analytic tools. For specifying the general solution, we use the matching with amplitudes $\bar{F}_{q, g}$ which describe the same Compton scattering but the external photon is real. As $\bar{F}_{q, g}$ do not depend on $y$, IREEs for them are algebraic, so they can easily be solved. The same is true concering IREEs for $f_{r r^{\prime}}$. Both IREEs for these auxiliary amplitudes and solutions to them can be found in Ref. [5]. 


\section{Expressions for $T_{q, g}$ in DLA}

Solving Eq. ([2.5), combining the solution and ([2.2), then using the Optical theorem (2.1) allows us to obtain expressions for $T_{q, g}$ in terms of the parton-parton amplitudes:

$$
\begin{aligned}
& T_{q}(\omega, y)=\int_{-1 \infty}^{1 \infty} \frac{d \omega}{2 \imath \pi}\left(w / \mu^{2}\right)^{\omega}\left[C_{(+)} e^{\Omega_{(+)}^{y}}+C_{(-)} e^{\Omega_{(-)}}\right] \\
& T_{g}(\omega, y)=\int_{-1 \infty}^{1 \infty} \frac{d \omega}{2 \imath \pi}\left(w / \mu^{2}\right)^{\omega}\left[C_{(+)} \frac{h_{g g}-h_{q q}+\sqrt{R}}{2 h_{q g}} e^{\Omega_{(+)} y}+C_{(-)} \frac{h_{g g}-h_{q q}-\sqrt{R}}{2 h_{q g}} e^{\Omega_{(-)} y}\right],
\end{aligned}
$$

where $h_{r r^{\prime}}=1 /\left(8 \pi^{2}\right) f_{r r^{\prime}}$

$$
\begin{gathered}
\Omega_{( \pm)}=\frac{1}{2}\left[h_{g g}+h_{q q} \pm \sqrt{R}\right] \\
R=\left(h_{g g}+h_{q q}\right)^{2}-4\left(h_{q q} h_{g g}-h_{q g} h_{g q}\right)=\left(h_{g g}-h_{q q}\right)^{2}+4 h_{q g} h_{g q} \\
C_{(+)}=e^{2 \frac{h_{q g} h_{g q}-\left(\omega-h_{g g}\right)\left(h_{g g}-h_{q q}-\sqrt{R}\right)}{2 G \sqrt{R}}} \\
C_{(-)}=e^{2} \frac{-h_{q g} h_{g q}+\left(\omega-h_{g g}\right)\left(h_{g g}-h_{q q}+\sqrt{R}\right)}{2 G \sqrt{R}}
\end{gathered}
$$

and

$$
G=\left(\omega-h_{q q}\right)\left(\omega-h_{g g}\right)-h_{g g} h_{q g} .
$$

Explicit expressions for $h_{r r^{\prime}}$ can be found in Ref. [5] and substituted in (B.D). Instead of doing so, we focus on consideration of the small- $x$ asymptotics of $T_{q, g}$.

\section{Small- $x$ asymptotics of $T_{q, g}$}

Applying the Saddle-Point method to Eq. (B.T) leads us to the asymptotics $T_{q, g}^{(a s)}$ at $x \rightarrow 0$. It is easy to obtain a similar asymptotics for $F_{1}$ substituting Eq. (B. II) in (ㅍ. II) and applying the Saddle-Point method to it at $x \rightarrow 0$, we arrive at the small $x$ asymptotics of $F_{1}$ :

$$
F_{1} \sim F_{1}^{(a s)}=\kappa\left(\frac{1}{x}\right)^{\omega_{0}}\left(\frac{Q^{2}}{\mu^{2}}\right)^{\omega_{0} / 2},
$$

where $\omega_{0}$ is the leading singularity, or intercept and $\kappa$ is the impact factor. The only difference between the asymptotics $T_{q, g}^{(a s)}$ and $F_{1}^{(a s)}$ is the difference in the impact factors. Eq. (4.]) states that asymptotically $F_{1}$ depends on the single variable $\zeta=Q^{2} / x^{2}$. We call Asymptotic Scaling this confluence of the $Q^{2}$ - and $x$ - dependence. The intercept $\omega_{0}$ was calculated in Ref. [\$] under various approximations. In particular, when the roughest approximation of fixed $\alpha_{s}$ and absence of quark contributions was used, the intercept $\omega_{H}$ was maximal while when $\alpha_{s}$ was running and quark and gluon contributions were accounted for, the intercept $\omega_{S}$ was minimal: 


$$
\omega_{H}=1.35, \omega_{S}=1.07 .
$$

Eq. (4.2) demonstrates that the Reggeon in (4. is is a supercritical Pomeron. Eq. (4. (1) is the asymptotics of the DL series (ㅍ.4), so the intercepts in (4.2) have nothing in common with ones of the BFKL Pomeron though $\omega_{H}$ is close to the intercept of the LO BFKL Pomeron and $\omega_{S}$ almost coincides with the intercept of the NLO BFKL Pomeron.

No doubt, the asymptotic expression $F_{1}^{(a s)}$ of Eq. (4.d) looks much simpler than the parent amplitudes $T_{q, g}$ of Eq. (B. (ل). It prompts to use $F_{1}^{(a s)}$ instead of $F_{q, g}$ at any $x$. However, the asymptotics should be used in their application region only. In order to evaluate such region we study numerically the $x$-dependence of $R_{(a s)}=T_{q}^{(a s)} / T_{q}$ at different $Q^{2}$. The fastest approach of $T_{q}$ to its asymptotics takes place when $Q^{2}=\mu^{2}$. This plot it shown in Fig. 1 .

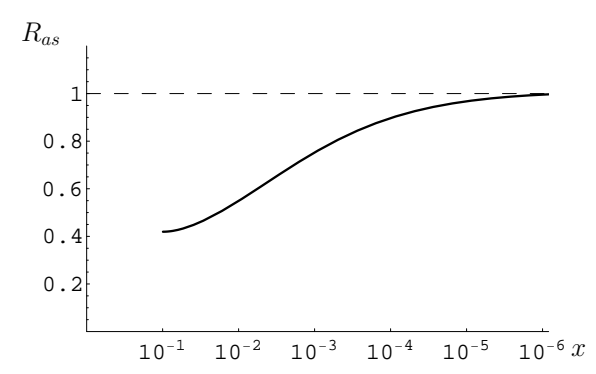

Figure 1: Approach of $T_{q}$ to its asymptotics $T_{q}^{a s}$ at $Q^{2}=\mu^{2}$.

The asymptotics represent reliably the parent amplitude when $R_{(a s)}$ is close to unity. Fig. 1 demonstrates that $R_{a s}=0.9$ at $x \approx 5.10^{-5}$ providing $Q^{2}=\mu^{2} \approx 1 \mathrm{Gev}^{2}$. When $Q^{2}$ grows, the approach of $R_{a s}$ to unity is slower. It drives us to conclude that the small- $x$ asymptotics, i.e. Pomerons, should not be used at $x>10^{-6}$ when $Q^{2} \approx 10 \mathrm{Gev}^{2}$ or greater.

\section{Conclusion}

We have calculated the perturbative component of $F_{1}$ in DLA. Applying the Saddle-Point method to the results obtained we calculated the small- $x$ asymptotics of $F_{1}$, which proved to be a supercritical Pomeron. In the literature, the Pomerons with the large intercepts like $\omega_{H}$ are addressed as hard Pomerons while smaller intercept like $\omega_{S}$ are attributed to the soft Pomerons. The hard and soft intercepts of Eq. (4.2) are not independent. They are related to the accuracy of calculations: The value of the intercept of this Pomeron decreasing with increase of the accuracy of calculations from $\omega_{H}$ down to $\omega_{S}$. The same is true for the BFKL Pomeron. So, the concept of independent hard and soft Pomeron does not agree with Perturbative QCD.

We think that introducing two or more Pomerons is caused by purely practical needs when the Pomerons are used well outside their applicability region instead of their parent amplitude. For example, studying $R_{a s}$ at $Q^{2}=10 \mathrm{GeV}^{2}$ yields that the impact of the Pomeron $R_{a s} \approx 0.6$ at $x=10^{-4}$ , i.e. the impact of the Pomeron is almost twice less than required. The theoretically correct way out of this situation is to use the parent amplitudes $T_{q, g}$ instead of the Pomeron. However, if one prefers 
to avoid it and to work with Pomerons, introducing an additional Pomeron becomes necessary, albeit without theoretical grounds.

We hope that in this talk we have given compelling reasons in favor of importance of the DL contribution to $F_{1}$ and its Pomeron asymptotics $F_{1}^{(a s)}$ for theory of DIS. These objects should be used in analysis of experimental data on a par with the BFKL Pomeron.

\section{References}

[1] G. Altarelli and G. Parisi, Asymptotic Freedom in Parton Language, Nucl. Phys. B 126 (1977) 297; V. N. Gribov and L. N. Lipatov, Deep inelastic e p scattering in perturbation theory, Sov. J. Nucl. Phys. 15 (1972) 438;

L. N. Lipatov, The parton model and perturbation theory, Sov. J. Nucl. Phys 20 (1972) 94;

Yu. L. Dokshitzer, Calculation of the structure functions for the Deep Inelastic scattering and e+e-annihilation by perturbation theory inquantum chromodynamics, Sov. Phys. JETP 46 (1977) 641.

[2] E. A. Kuraev, L. N. Lipatov, and V. S. Fadin, Multi-Reggeon processes in Yang-Mills theory, Sov. Phys. JETP 44 (1976) 443;

E.A. Kuraev, L. N. Lipatov, and V. S. Fadin, The Pomeranchuk singularity in non abelian gauge theories, Sov. Phys. JETP 45 (1977) 199;

I. Balitsky and L. N. Lipatov, The Pomeranchuk singularity in Quantum Chromodynamics, Sov. J. Nucl. Phys. 28 (1978) 822.

[3] V. S. Fadin and L. N. Lipatov, BFKL Pomeron in the next-to-leading approximation, Phys. Lett. B 429 (1998) 127;

G. Camici and M. Ciafaloni, Energy scale(s) and next-to-leading BFKL equation, Phys. Lett. B 430 (1998) 349.

[4] Stanley J. Brodsky, V. S. Fadin, V. T. Kim, L. N. Lipatov, G. B. Pivovarov, The QCD Pomeron with optimal renormalization, JETP Lett. 70 (1999) 155.

[5] D. I. Ermolaev, S. I. Troyan, Singlet structure function $F_{1}$ in double-logarithmic approximation, EPJC 78 (2018) 204.

[6] B.I. Ermolaev, M. Greco, S.I. Troyan, Infrared Evolution Equations: Method and applications, Acta Phys. Polon. B 38 (2007) 2243-2260. 